\title{
Large low frequency dielectric constant exhibited by hydrated rock materials
}

\author{
By Apostolos Kyritsis, $\left.{ }^{*}, * *\right)$ Maria Siakantari, ${ }^{*)}$ Aglaia Vassilikou-Dova, ${ }^{*)}$ Polikarpos Pissis, ${ }^{* *)}$ \\ and Panayoitis VAROTSOS ${ }^{*, \dagger)}$ \\ (Communicated by Seiya UyEDA, M.J.A., Feb. 13, 2001)
}

\begin{abstract}
The dielectric properties of low porosity polycrystalline marble and granite rocks were investigated using broadband dielectric relaxation spectroscopy $\left(10^{-2}-10^{6} \mathrm{~Hz}\right)$ at fixed room temperature $(295 \mathrm{~K}$ ). The water content of the specimens was varied between 0 and $0.22 \%$ (mass of absorbed water per mass of dry rock material \%). The dielectric relaxation spectra were found to exhibit large low-frequency dispersion that depends strongly on the water content. A thorough study of this dispersion reveals that the main part of the enhanced $\varepsilon^{*}$ values originates from bulk effects.
\end{abstract}

Key words: Dielectric properties; rocks; low frequency dispersion.

Introduction. The dielectric spectra of clay, water- or brine-saturated rocks exhibit large complex permittivity values at low frequencies, $f,\left(f<10^{6} \mathrm{~Hz}\right)$ although neither rock nor water are dispersive in that frequency range. ${ }^{1)-5)}$ The values of the complex permittivity, $\mathcal{E}^{*}$, for the rock-water composite (usually of the order of $10^{5}-10^{6}$ ) exceed by far those of both constituents. Numerous investigations on water saturated rocks showed that upon variation of the conductivity of water, the dielectric permittivity of rocks is scaled accordingly. ${ }^{2), 6), 7)}$ Knight and co-workers have studied in detail the dependence of dielectric properties of rocks on their hydration level; they found that the creation of a thick water layer within the pores of the rock matrix affects significantly the dielectric properties of the rocks, controlling the transition from short to long range mobility of the charge carriers. ${ }^{8), 99}$

In this paper we present results of dielectric measurements on natural, low porosity (porosity $<1 \%$ ) polycrystalline rocks, coming from Greece; granite from Kavala district and marble from Ioannina district. The dielectric properties were studied at several hydration levels, varied from dry (water content $h \approx 0 \%$ gr. of water per gr. of dry material) to highly hydrated specimens (h $\approx 0.25 \%$ ). To achieve the various hydration levels, the specimens were allowed to equilibrate in various dessi-

\footnotetext{
*) Athens University, Physics Department, Section of Solid State Physics, Panepistimiopolis, 15784 Zografos, Greece.

**) National Technical University of Athens, Department of Physics, Zografou Campus, 15780 Athens, Greece.

$\dagger$ Correspondence to: P. Varotsos.
}

cators, where the relative humidity $(\mathrm{RH})$ was monitored between 0.06 and 0.98 using different saturated salt solutions. We emphasis that throughout this study the hydration procedure was restricted to hydration of rocks only in atmospheres with $\mathrm{RH}<1$, i.e., the specimens were not exposed over water or immersed in water to absorb water molecules. If $\mathrm{RH}=1$, the absorbed water molecules may form separate bulk water phase within the host matrix, ${ }^{10)}$ which could affect drastically the dielectric behaviour of the mixture. When $\mathrm{RH}<1$, we prevent the creation of a separate bulk water phase without excluding the creation of water clusters. Thus we investigate mainly the role of waterrock interactions on the dielectric properties of hydrated rocks excluding possible significant contribution from a separate highly conductive water phase. Further experiments on rock samples hydrated in atmospheres with $\mathrm{RH}=1$, i.e., over water or immersed in water, reveal that the conductivity of the specimens in that case is drastically increased showing almost no frequency dispersion (features of a dc conductivity). Therefore, the comparative study of the dielectric properties of the specimens hydrated in atmospheres with $\mathrm{RH}<1$ and $\mathrm{RH}=1$ support the assertion that the hydration conditions in the present study do not favour the creation of an extended water phase within the rock matrix.

Dielectric susceptibility measurements were carried out at room temperature $(\mathrm{T}=295 \mathrm{~K})$ in the frequency range $10^{-2}$ to $10^{6} \mathrm{~Hz}$. Two measurement systems were used: 1) A Solatron-Schlumberger frequency response analyzer FRA1260 with a buffer amplifier of variable gain 
and working frequency $10^{-2}-10^{6} \mathrm{~Hz}$. 2) A HewlettPackard precision LCR meter, 4284A, with working frequency range $20-10^{6} \mathrm{~Hz}$. Admittance measurements $Y^{*}(f)$ were performed and the following relation holds:

$$
\varepsilon^{*}(f)=\frac{Y^{*}(f)}{i 2 \pi f C_{0}}
$$

where $C_{0}$ is the vacuum capacitance of the measuring cell. ${ }^{11,12)}$ The complex impedance, $Z^{*}$, is estimated according to the relation:

$$
Z^{*}=\frac{1}{Y^{*}}=Z^{\prime}+i Z^{\prime \prime}
$$

The present paper is focused on the large low frequency dielectric dispersion of hydrated rocks and investigates the nature (electrode/sample interface or intrinsic phenomenon) of that low frequency dispersion. Several formalisms provided by dielectric relaxation spectroscopy method, that lead to a deeper insight into the underlying microscopic mechanisms, which give rise to the high $\varepsilon^{*}$ values observed, are presented elsewhere. ${ }^{11), 12)}$

Overall dielectric response. Fig. 1 shows the frequency dependence of the real $\boldsymbol{\varepsilon}^{\prime}$ (a) and the imaginary $\varepsilon^{\prime \prime}$ (b) part of $\varepsilon^{*}$, for a marble specimen, at fixed temperature $295 \mathrm{~K}$ and for five different water contents. High values of both, $\varepsilon^{\prime}$ and $\varepsilon^{\prime \prime}$, were measured, especially at low frequencies. Additionally, these figures demonstrate the strong water content dependence of $\varepsilon^{*}(f)$ spectra. For a dry specimen $(\mathrm{h}=0.005 \%)$, the $\varepsilon^{\prime}(f)$ plot in Fig. 1 shows no dispersion, while the $\varepsilon^{\prime \prime}(f)$ plot exhibits an increase of $\varepsilon^{\prime \prime}$ values with decreasing frequency, probably because of the mobility of intrinsic charge carriers. Upon gradually increasing $h$, the $\varepsilon^{\prime}$ and $\varepsilon^{\prime \prime}$ values increase significantly in the whole frequency range studied, thus indicating that the absorbed water molecules contribute to the mechanisms responsible for the large low-frequency dispersion.

According to effective medium theories, the $\boldsymbol{\varepsilon}^{\prime}$ values may depend significantly on the morphology of the mixture (geometric factors) and the specific rockwater interactions (physicochemical factors). ${ }^{4), 5), 9)}$ These assertions are supported by the comparison of the $\mathrm{h}$ dependence of $\boldsymbol{\varepsilon}^{\prime}$ for marble and granite. Applying similar hydration procedure, granite absorbs more water at saturation, $h_{\max }$, than marble. Note that $h_{\max }$ is defined to be the water content, obtained by keeping the specimens for 7 days over $\mathrm{K}_{2} \mathrm{SO}_{4}$ salt solution in a sealed jar $(\mathrm{RH}=$ 0.98 ). We found $h_{\text {GRAmax }}=0.22 \%$ for granite whereas $\mathrm{h}_{\text {MARmax }}=0.08 \%$ for marble. However, comparing $\mathcal{\varepsilon}^{\prime}$ values versus the scaled water content $h / h_{\text {amb }}, h_{\text {amb }}$ being the (a)

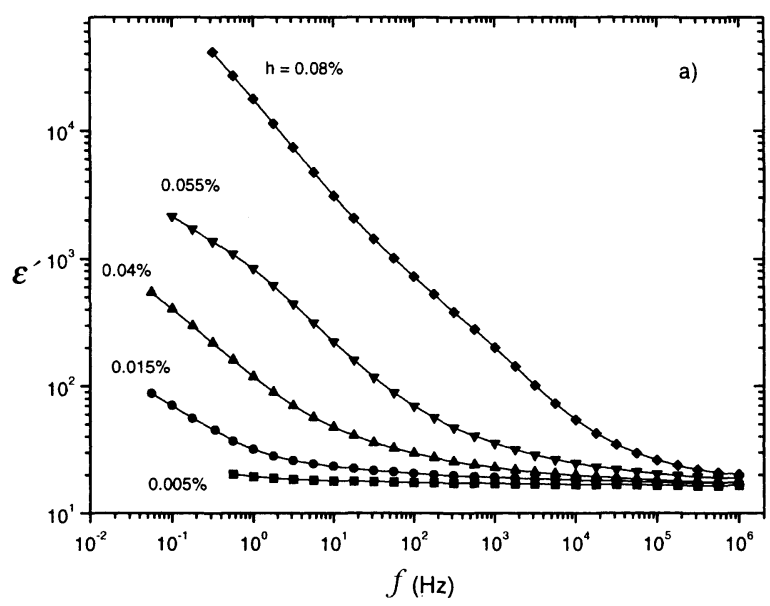

(b)

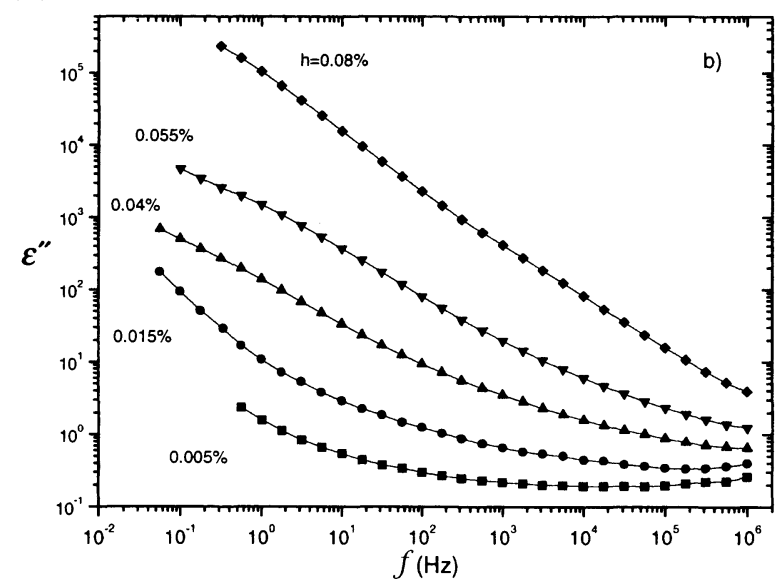

Fig. 1. Real part, $\varepsilon^{\prime}(\mathrm{a})$ and imaginary part, $\varepsilon^{\prime \prime}(\mathrm{b})$ of the complex dielectric permittivity, $\varepsilon^{*}$, vs frequency for a marble specimen at several water contents given in the plots. The curves have been drawn as a guide to the eye.

water content at ambient condition $(\mathrm{RH} \approx 0.50$ and $\mathrm{T}=$ $297 \mathrm{~K}$ ), the two rock systems exhibit comparable maximum values for the dielectric constant. The value of $h_{\text {amb }}$ is $h_{\text {GRAamb }}=0.11 \%$ for granite and $h_{\text {MARamb }}=0.04 \%$ for marble. Therefore, we conclude that it is not the absolute value of the water content that controls the dielectric behaviour of rock-water system, but the organization of absorbed water molecules within the rock matrix and the specific rock-water interaction existed. Furthermore $h_{\text {amb }}$ is found to be a characteristic water content level for the rock matrix, in the sense that the dielectric properties of the mixture rock-water at each water content depend on the divergence of that water content level from $h_{\text {amb }}$.

Discrimination between bulk and electrode/ 
(a)

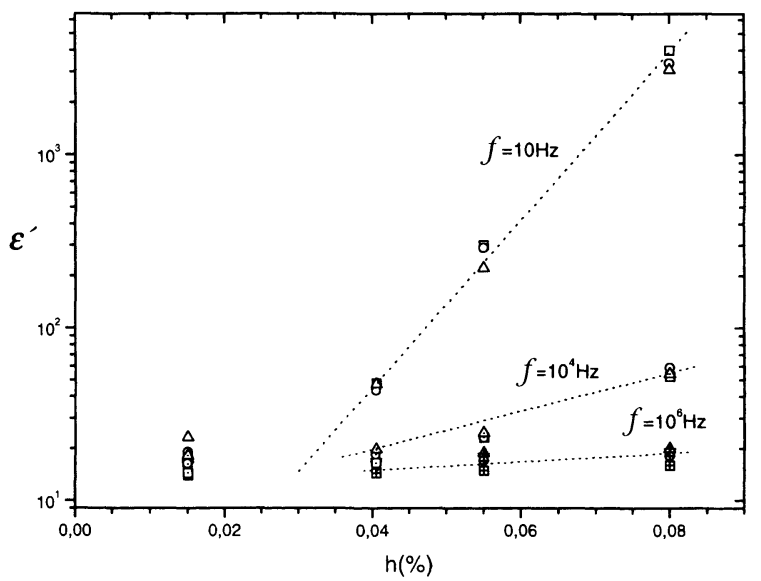

(b)

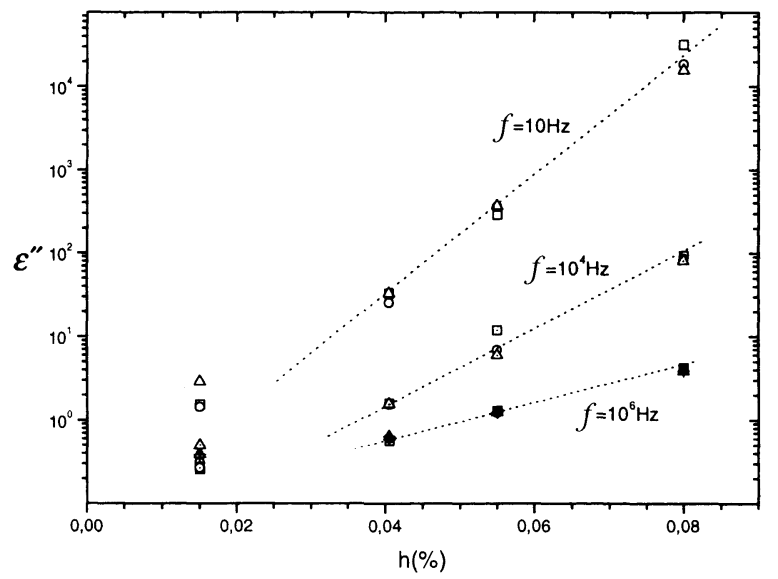

Fig. 2. Water content dependence of $\varepsilon^{\prime}(\mathrm{a})$ and $\varepsilon^{\prime \prime}(\mathrm{b})$ for three marble specimens with different thickness, $d=3.98 \mathrm{~mm}$ (squares), $5.68 \mathrm{~mm}$ (circles) and $7.15 \mathrm{~mm}$ (triangles), at three selected frequencies, $f=10 \mathrm{~Hz}$ (open symbols), $10^{4} \mathrm{~Hz}$ (dotted symbols) and $10^{6} \mathrm{~Hz}$ (cross centered symbols).

specimen interface effects. We now turn to the question, whether the large low-frequency $\varepsilon^{*}$ values constitute a characteristic property of the material (bulk effect) or originate from polarization effects at the electrode/specimen interface and, thus, depend mainly on the experimental conditions.

First, experiments with different electrode materials have been performed. Metal electrodes were used, which were kept in contact with the specimen by spring load, like gold, brass, stainless steel and aluminium foils (thickness of about $12 \mu \mathrm{m}$ ). In addition, sputtered aluminium and silver paste have also been used as electrodes and insulating low density polyethylene sheets as well. The experiments show, on one hand, that the electrode materials used show different electrical
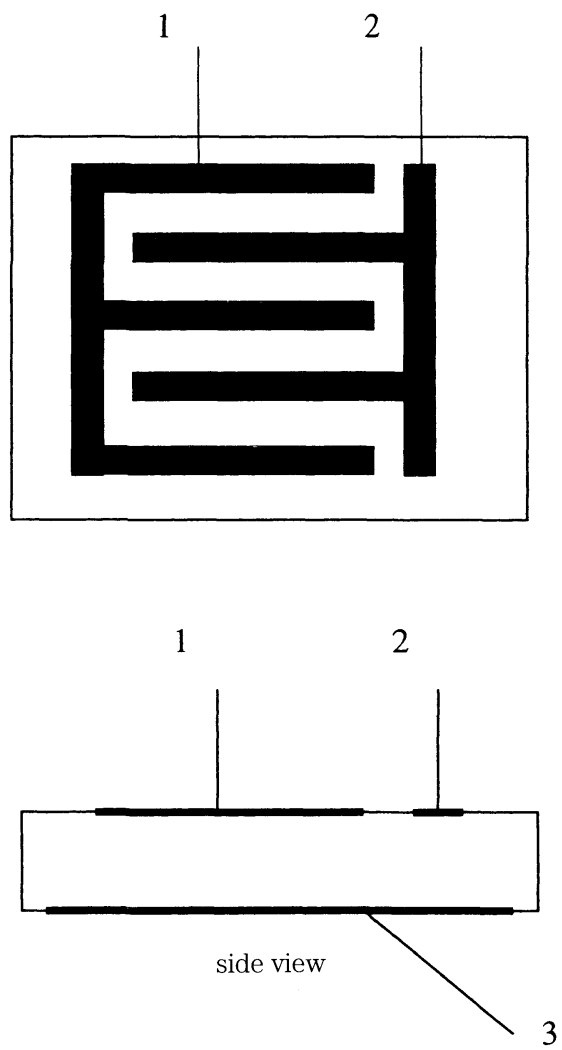

Fig. 3. Scheme of the electrode configuration for "surface" (comb-like electrodes) and "bulk" measurements (see the text).

behaviour, exhibiting features of totally blocking up to almost non blocking electrical character, and, on the other hand, that the whole $\mathcal{E}^{*}(f)$ spectrum is sensitive to the charge compensation ability of the electrode/sample interface. ${ }^{11)}$ This comparative study of electrode materials suggests that the sputtered aluminium electrodes have the less blocking character (among the aforementioned electrodes), i.e., charge transfer and compensation occur at the electrode and the additional impedance created at the electrode/specimen interface, if any, is of low magnitude. Therefore, all the dielectric measurements were performed on specimens with aluminium sputtered electrodes.

Second, in order to discriminate between bulk and electrode effects, dielectric experiments on specimens with different thickness were carried out. Dielectric measurements have been performed on marble specimens with three different thickness $(\mathrm{d}=3.98,5.68$ and $7.15 \mathrm{~mm}$ ) at four water contents. The $\varepsilon^{\prime}$ and $\varepsilon^{\prime \prime}$ values as function of $\mathrm{h}$ at three selected frequencies, $f=10,10^{4}$ and $10^{6} \mathrm{~Hz}$, are presented in Fig. 2 and show that for the whole frequency range both $\varepsilon^{\prime}$ (Fig. 2a) and $\boldsymbol{\varepsilon}^{\prime \prime}$ (Fig. 2b) 
(a)

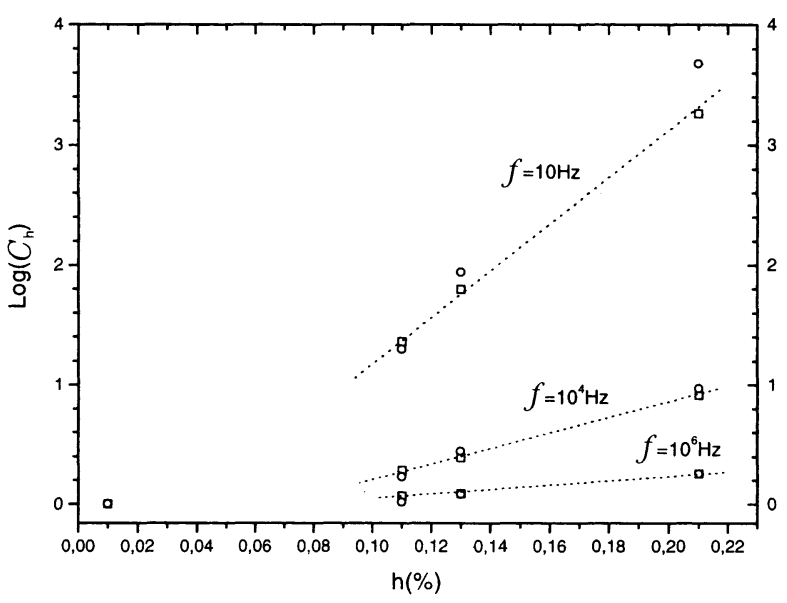

(b)

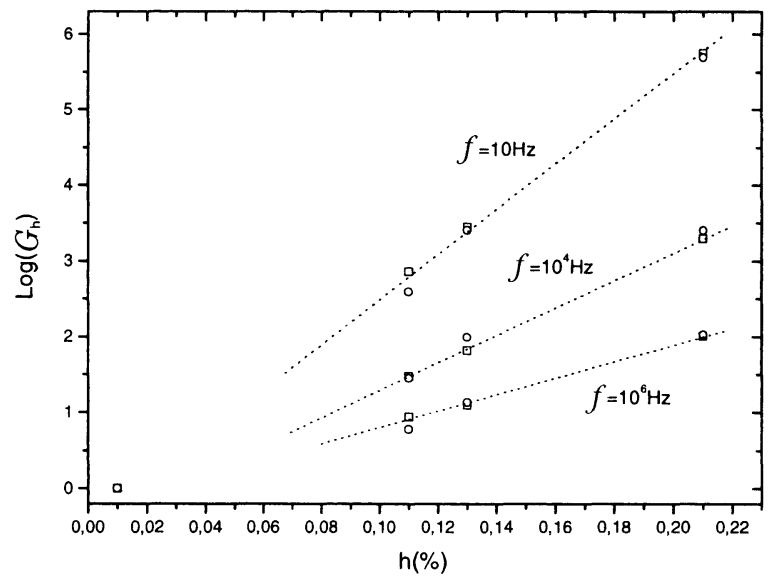

Fig. 4. Water content dependence of the logarithm of the ratios $C_{\mathrm{h}}$ (a) and $G_{\mathrm{h}}$ (b) for "bulk" (squares) and "surface" (circles) measurements, at three selected frequencies given in the plots. Details concerning $C_{\mathrm{h}}$ and $G_{\mathrm{h}}$ are given in the text.

are independent of the specimen's thickness. Hence, bulk effects must be responsible ${ }^{13)}$ for the main part of these high permittivity values. The data in Fig. 2 suggest also the existence of a critical water content, $h_{c}$, with $h_{c}=$ $0.04 \%$ (water content of marble at ambient conditions): for $\mathrm{h} \leq \mathrm{h}_{\mathrm{c}}$ a slight increase of $\boldsymbol{\varepsilon}^{*}$ values with increasing $\mathrm{h}$ is observed, whereas for $h \geq h_{c}, \mathcal{E}^{\prime}$ and $\varepsilon^{\prime \prime}$ values increase rapidly with h, i.e., the slope of $\varepsilon^{\prime}(\mathrm{h})$ and $\varepsilon^{\prime \prime}(\mathrm{h})$ curves is much higher for $h \geq h_{c}$, as compared to that for $\mathrm{h} \leq \mathrm{h}_{\mathrm{c}}$.

Third, using the proper electrode configuration, the dielectric experiments offer the possibility to study in parallel the bulk and surface dielectric properties. In Fig. 3 the electrode configuration for "bulk" and "surface"

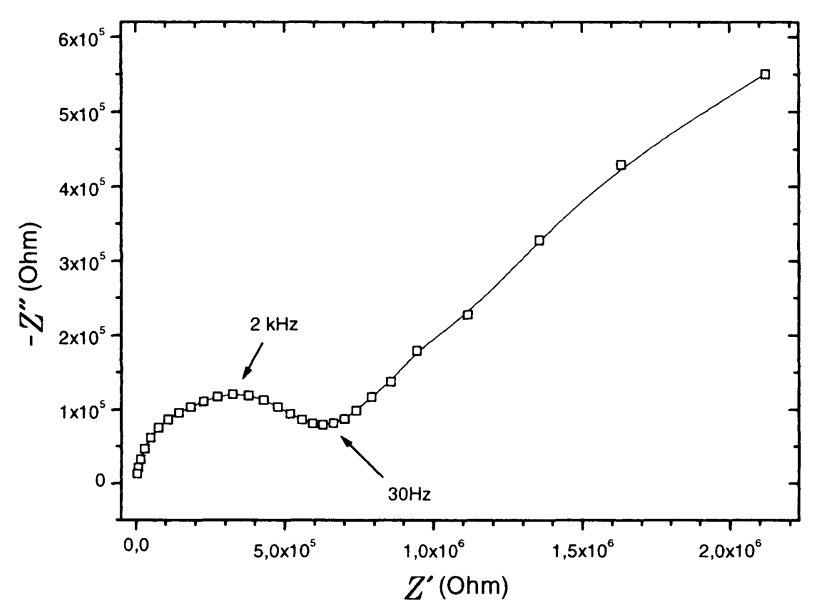

Fig. 5. $-Z^{\prime \prime}$ vs $Z^{\prime}$ plot for a marble specimen at $\mathrm{h}=0.08 \%$, the maximum water content in Fig. 1. The curve has been drawn as a guide to the eye.

(comb-like electrodes) measurements ${ }^{11)}$ is depicted. For "surface" dielectric susceptibility measurements the electrodes 1 and 2 being on the same surface of the specimen are the two active electrodes, whereas for "bulk" measurements electrode 3 is the high electrode while 1 and 2 are interconnected and work as the low electrode, i.e., the two active electrodes are on opposite surfaces of the specimen. Such an experiment has been performed in a granite specimen, at four water contents, $\mathrm{h}=0.01,0.11,0.13$ and $0.31 \%$ : the capacitance, $C$, and the conductance, $G$, were measured for two different electrode configurations; the "bulk" and the "surface" configuration. Fig. 4 presents the plots $\log \left(C_{\mathrm{h}}\right)$ (Fig. 4a) and $\log \left(G_{\mathrm{h}}\right)$ (Fig. 4b), for three selected frequencies, $f=10$, $10^{4}$ and $10^{6} \mathrm{~Hz}$, vs water content, h. The quantity $C_{\mathrm{h}}$ is defined as the ratio of the capacitance at any $h$ to the capacitance of the "dry" specimen $(\mathrm{h}=0.01 \%)$, i.e., $C_{\mathrm{h}}=$ $C(\mathrm{~h}) / C(\mathrm{~h}=0.01 \%)$, measured by each electrode configuration; as for $G_{\mathrm{h}}$, this stands for the corresponding ratio of the conductance values. Fig. 4 clearly depicts the similar h dependence of the "bulk" and the "surface" complex admittance at the three frequencies selected. This similarity obtained by these two experiments indicates that there is no additional contribution to the $\varepsilon^{*}$ data of any polarization mechanism on the surface of the specimens with increasing $h$. Our results suggest that the surface conductivity is dominated by the properties of the material between the electrodes 1 and 2, being the equivalent of "bulk" conductivity in three-dimensional process. There is no indication of an electrical behaviour being dominated by the response of a conduction mechanism spatially constrained on the surface, as 
found in other analogous experiments on mica, glass and sand materials. ${ }^{14)}$

Finally, by using the complex impedance plots $\left(-Z^{\prime \prime}\right.$ vs $\left.Z^{\prime}\right)$ it is possible to separate bulk from surface phenomena due to the fact that electrode polarization is characterized by larger relaxation times than the polarization mechanisms in the bulk. ${ }^{11), 12)}$ In Fig. 5 we show the $-Z^{\prime \prime}$ vs $Z^{\prime}$ plot for the marble specimen with thickness $\mathrm{d}=7.15 \mathrm{~mm}$ at the maximum water content $\left(\mathrm{h}_{\text {MARmax }}=\right.$ $0.08 \%)$. A separate arc of semicircle is clearly observed at high frequencies. In terms of the $Z^{*}$ formalism the "bulk" and "surface" effects are separated at the frequency region near $30 \mathrm{~Hz}$. The semicircle in the frequency region of $30-10^{6} \mathrm{~Hz}$ corresponds to polarization mechanisms in the bulk, whereas for $f<30 \mathrm{~Hz}$ specimen/electrode interface effects dominate, which are characterized by larger relaxation times. Bearing in mind that with increasing $h$ the electrode polarization mechanism becomes faster i.e., the corresponding relaxation time decreases, we conclude that for lower water contents $(h<0.08 \%)$ the electrode polarization mechanism shifts to lower frequencies, thus the frequency which separates bulk from electrode-sample interface effects shifts to lower values (lower than 30 $\mathrm{Hz})$.

Conclusion. The experimental findings suggest that for hydrated polycrystalline rock materials (marble and granite), the high permittivity values originate mainly from bulk effects; in other words, these materials are characterized by large dielectric constants and this feature is not an artificial one. (We have found that electrode polarization effects have a significant contribution to dielectric permittivity only for high water contents and then only for frequencies lower than about $10 \mathrm{~Hz}{ }^{11)}$ ) This assertion is further supported by additional dielectric measurements ${ }^{11), 12)}$ using the so called "guard ring". Tentative models for the explanation of the large low-fre- quency dispersion of the dielectric constant have been discussed elsewhere. We note that the dielectric behaviour of hydrated rock specimens shows similarities with the behaviour exhibited by materials with high degree of disorder or by other water containing systems. ${ }^{15)}$ This large low frequency dispersion, deviating strongly from the conventional behaviour exhibited by systems that obey Debye-like dynamics, is the subject of many experimental investigations and theoretical models.

Acknowledgments. A. K. gratefully acknowledges financial suport from the Greek State Scholarships Foundation. We are also thankfull to Prof. B. Hadjikontis for providing the samples.

\section{References}

1) Olhoeft, G. R. (1985) Geophysics 50, 2492 -2503.

2) Lockner, D. A., and Byerlee, J. D. (1985) J. Geophys. Res. 90, 7837-7847.

3) Hilfer, R. (1993) Physica A 194, 406-414.

4) Gueguen, Y., and Palciauskas, V. (1994) Introduction to The Physics of Rocks. Princeton University Press, Princeton.

5) Thevanayagam, S. (1997) J. Appl. Phys. 82, 2538-2547.

6) Kenyon, W. E. (1984) J. Appl. Phys. 55, 3153-3159.

7) Nettelblad, B., and Niklasson, G. A. (1994) Solid State Communications 90, 201-204.

8) Knight, R. J., and Nur, A. (1987) Geophysics 52, 644-654.

9) Knight, R. J., and Endres, A. (1990) Geophysics 55, 586-594.

10) Ribelles, J. L. G., Pradas, M. M., Ferrer, G. G., Torres, N. P., Gimenez, V. P., Pissis, P., and Kyritsis, A. (1999) J. Polym. Sci., Part. B: Polym. Phys. 37, 1587-1599.

11) Kyritsis, A., Siakantari, M., Vassilikou-Dova, A., Pissis, P., and Varotsos, P. (Part I) (in preparation).

12) Kyritsis, A., Siakantari, M., Vassilikou-Dova, A., Pissis, P., and Varotsos, P. (Part II) (in preparation).

13) Nettelblad, B. (1996) J. Appl. Phys. 79, 7106-7113.

14) Jonsher, A. K. (1996) Universal Relaxation Law. Chelsea Dielectric Press, London.

15) Niklasson, G. A. (1989) Physica D 18, 206-265. 